\title{
Two dimensional echocardiographic assessment of aortic valve morphology: feasibility of bicuspid valve detection Prospective study of 100 adult patients
}

\author{
MICHAEL J ZEMA, MICHELE CACCAVANO \\ From the Division of Cardiology, Department of Medicine, Brookhaven Memorial Hospital Medical Center, Patchogue, \\ New York, USA
}

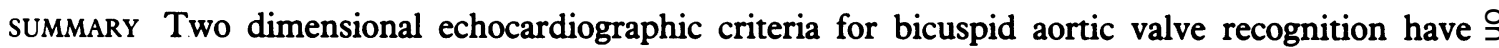
greater specificity than previously proposed $\mathbf{M}$-mode echocardiographic criteria. The potential clinical use of the two dimensional technique is, however, limited by the technical inability to image adequately the aortic valve leaflets in many patients. One hundred consecutive adult patients undergoing two dimensional echocardiography were prospectively studied. Valve cusp number could not be determined because of dense calcification in eight patients. A bicuspid aortic valve was diagnosed in a single subject. A parasternal short axis view disclosed three commissures (diastolic "Y" configuration) in only 26 patients. Technically adequate parasternal short axis imaging was more likely in younger ! patients and in non-smokers. In patients not successfully imaged from the parasternal approach, an anteriorly tilted apical four chamber view showed a diastolic " $Y$ " configuration in an additional eight cases. Considering the high prevalence in our population of incomplete two dimensional echocardiographic aortic valve leaflet imaging, angiographic and/or pathological studies must be performed to establish the correlation between these incomplete echocardiographic patterns and aortic valve anatomy if two dimensional echocardiography is to have widespread application in the diagnosis of the congenital bicuspid aortic valve.

With the exception of mitral valve prolapse, the bicuspid aortic valve is the most common congenital heart lesion found in adults, seen in $0.7 \%$ to $2 \cdot 0 \%$ of routine necropsies. ${ }^{12}$ Moreover, unlike the usually excellent prognosis associated with mitral valve prolapse ${ }^{3}$ the subsequent complications of a bicuspid aortic valve include infective endocarditis, ${ }^{4-7}$ progressive valvular stenosis, and/or valvular regurgitation. ${ }^{8-12}$ Fenoglio and coworkers ${ }^{7}$ have suggested that about one-third of patients over the age of 20 years born with a non-stenotic bicuspid aortic valve will develop aortic stenosis, one-third will develop aortic regurgitation (in most cases because of endocarditis), and the remainder will be free of any haemodynamically significant problem. The greatest single cause of death in subjects with a bicuspid aortic valve is infective endocarditis, with over $75 \%$ of these deaths occurring before the age of $50 .^{7}$

Two dimensional echocardiography appears to be

Accepted for publication 26 May 1982

more specific than M-mode echocardiography in the $\overparen{\mathbb{\Phi}}$ diagnosis of bicuspid aortic valve. 1314 Practical application of two dimensional echocardiography to this problem, however, requires that technically adequate images of the aortic valve cusps be obtained.

The objectives of this prospective study were, there. fore, (1) to determine the frequency with which the 음 commissural margins of the aortic valve cusps could bo $D$ imaged by two dimensional echocardiography, (2) to determine the factors affecting the technical feasibility $N$ of the above determination, and (3) to evaluate the usi of an anteriorly tilted apical four chamber view fo $N$ imaging the aortic valve commissural margins is $\omega$ diastole in certain subjects without an adequat parasternal echocardiographic window.

\section{Methods}

(A) PATIENT SELECTION

One hundred (43 men and 57 women) consecuti adult patients aged 18 to 91 years (mean 54 years), wit no previous history of aortic valve replacement, wel 
Table Age distribution of patients studied

\begin{tabular}{lc}
\hline $\begin{array}{l}\text { Age group } \\
(y)\end{array}$ & No. of patients \\
\hline $18-29$ & 9 \\
$30-39$ & 14 \\
$40-59$ & 35 \\
$60-91$ & 42 \\
Total & 100 \\
\hline
\end{tabular}

referred to the Division of Cardiology, Brookhaven Memorial Hospital Medical Center, for two dimensional echocardiography. The age distribution is shown in the Table. The clinical indication for the echocardiographic examination covered a wide spectrum of cardiac disease. Though certain patients were referred with diagnoses of aortic stenosis and/or regurgitation, none was suspected of having a congenital bicuspid aortic valve in particular on clinical grounds alone. Patients who had smoked the equivalent of 20 cigarettes a day for 10 years or more were classified as smokers. Forty-nine patients were smokers and 51 were non-smokers. Weight in relation to height (ponderal index) [height (in) $/ \sqrt{\text { weight (lb) }}$ ranged from $9 \cdot 2$ to 13.9 (mean $11 \cdot 9$ ).

\section{(B) EQUIPMENT}

Ultrasound examination was performed with a commercially available mechanical two dimensional imaging system (Picker Echoview System 80CI, Northford, Conn.) with a $2.25 \mathrm{MHz}$ transducer imaging 30 and 60 degree sectors. Image rates were 45 and 60 images per second when using the 60 and 30 degree sectors, respectively. The wide angle was useful for spatial orientation and aortic valve plane localisation, while the 30 degree angle provided better image quality and structural detail. Images were recorded on a 0.5 in videotape via a Sanyo VTC 1000 cassette recorder (Sanyo Corporation, Compton, CA) for analysis, in real time, slow motion, and single frame format. Individual frames were photographed on Polaroid film (Polaroid Corporation, Cambridge, Ma).

\section{(C) TECHNIQUE}

All examinations were performed in the supine and left lateral decubitus positions by one of the authors (MZ). Parasternal short axis views of the heart and great arteries were obtained by directing the beam in a plane perpendicular to the long axis of the left ventricle and tilting the transducer superiorly. An apical four chamber view was obtained by placing the transducer at the point of maximal impulse, with the patient in the left lateral decubitus position and directing the ultrasonic beam from apex to base, perpendicular to the plane of the atrial and ventricular septa. On tilting the transducer slightly more anteriorly, the aortic valve could be recorded. ${ }^{15}$ Visualisation of aortic leaflet commissures by this latter technique was systematically attempted only in subjects in whom adequate parasternal short axis imaging was not technically possible. The aortic valve was examined from multiple interspaces and the transducer carefully swept through the entire space since cardiac excursion often temporarily removed the aortic valve from the plane of visualisation. Both parasternal short axis and tilted apical four chamber echocardiographic views could often be recorded more easily with held expiration; in some patients, however, apical images were obtained best during held inspiration. Real time visualisation of valve motion was useful, inasmuch as it was easier to recognise structures and their interrelations as they moved through cyclical excursion than if seen only in static display. The static images, moreover, were degraded optically because stop action pictures show only a single field of a complete two field video frame. The aortic root images were reviewed for location of commissural edges, number of cusps visualised, and shape of the aortic valve orifice in systole. Still frames for display as well as data for statistical analyses were derived only from diastolic echocardiographic images.

\section{(D) STATISTICS}

The two tailed Student's t test was used to determine the significance of differences in mean \pm standard deviations between two groups of subjects. The effect of certain modifying factors upon adequate visualisation of aortic valve leaflets was determined using $\chi^{2}$ test uncorrected for continuity. ${ }^{16}$ Multiple regression analyses were not performed. An alpha level of 0.05 was established as the level of significance.

\section{Results}

\section{(A) PARSTERNAL SHORT AXIS VIEW}

Two dimensional echocardiography disclosed three apparent commissures (diastolic "Y" configuration) (Fig. 1A) in 26 patients, suggesting a tricuspid aortic valve. ${ }^{13} 15$ Two commissures (diastolic "V" configuration) (Fig. 1B and C) were imaged in an additional 25 subjects. Only a single commissure ending at a central nodulus Arantii was seen in diastole in four cases (Fig. 2A). Either two or three sides of the previously described "inverted triangle configuration"15 (Fig. 3 systole) were noted in systole in all the above cases. One patient had a bicuspid aortic valve diagnosed by both systolic and diastolic leaflet configuration ${ }^{13}{ }^{15}$ (Fig. 4A and B), and had an aortic ejection sound on phonocardiography and only two sinuses of Valsalva visible on supravalvular aortography.

(B) FACTORS AFFECTING TECHNICAL FEASIBILITY OF PARASTERNAL SHORT AXIS VIEW In 73 of the 100 patients, determination of the number of cusps was not possible because (1) failure to obtain 


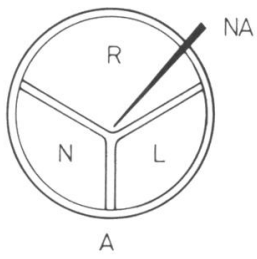

$(n=26)$
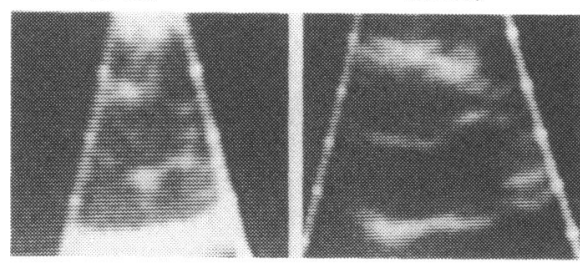

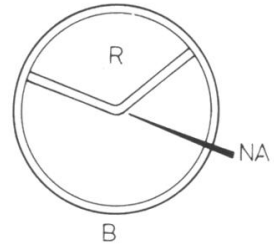

ln $n=24$

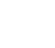

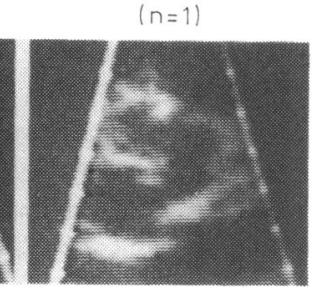

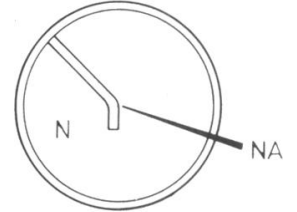

Fig. 1 (A) Parasternal short axis view diastolic frame showing three aortic cusps (" $Y$ "

configuration); $(B)$ and $(C)$ parasternal short axis view diastolic frames showing " $V$ " configuration (two aortic leaflet commissures). $R$, right coronary cusp; $N$, non-coronary cusp; $L$, left coronary cusp; $N A$, noduli Arantii.

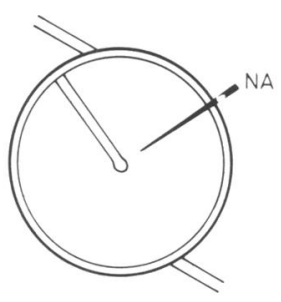

A

$(n=4)$

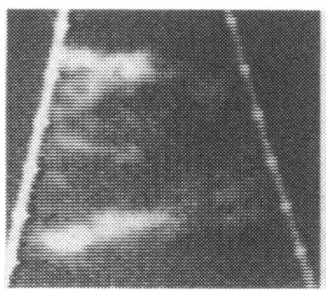

Fig. 2 (A) Parasternal short axis view diastolic frame showing only a single commissure. NA, nodulus Arantii; $(B)$ parasternal short axis view diastolic frame showing highly calcified aortic valve cusps.

an adequate tomographic short axis view at the level of the aortic valve in 36 patients; (2) though a technically adequate tomographic section was obtained optimal visualisation not realised in 29 patients (Fig. 1B, 1C, and $2 \mathrm{~A}$ ); and (3) the valve cusps densely calcified in eight patients (Fig. 2B). Technically adequate parasternal short axis imaging was more likely in nonsmokers $(37 / 51(73 \%)$ vs $27 / 49(55 \%)(\mathrm{p}<0.05))$ and in younger patients $(48.9 \pm 18.0$ y vs $57.2 \pm 13.0$ y) $(p<0.05)$. Sex $(37 / 64(58 \%)$ women vs $20 / 36(56 \%)$ women (NS)) and weight in relation to height $(12 \cdot 1 \pm 1 \cdot 0)$ vs $(11 \cdot 9 \pm 0 \cdot 8)(\mathrm{NS})$ were not factors.
A

Equal cusps and commissures

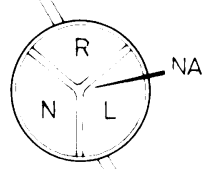

Diastole

B

Unequal cusps and equal commissures

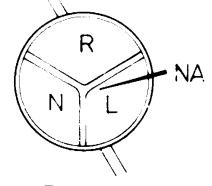

Diastole

C Unequal cusps and commissures

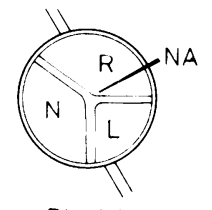

Diastole

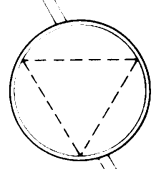

Systole

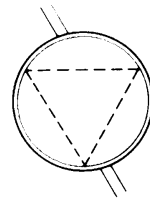

Systole

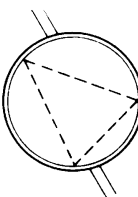

Systole
Fig. 3 Diagram of equal and unequal sized aortic valve cusps; on the right the aortic valve is opened. $R$, right coronary cusp; $N$, non-coronary cusp; L, left coronary cusp; NA, noduli Arantii.

(C) ANTERIORLY TILTED APICAL FOUR CHAMBER VIEW

In 40 subjects in whom maximally only one aortic leaflet commissure was imaged from the parasternal echocardiographic window, an anteriorly tilted apical four chamber view disclosed a diastolic " $Y$ " configuration in eight (Fig. 5A), suggesting a tricuspid aortic valve. A diastolic " $V$ " configuration with visualisation of only two commissures was seen in five subjects (Fig. 5B). In eight additional subjects only a partial " $V$ " configuration was imaged. The noduli Arantii and very central portions of the commissural edges of the valve 


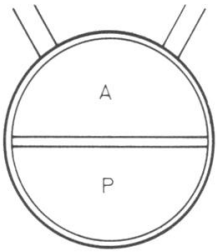

A

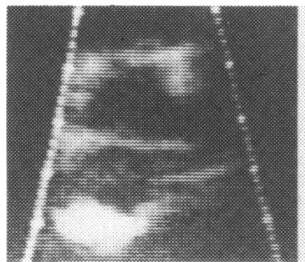

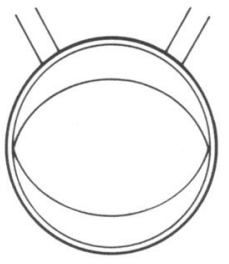

$B$

Systole

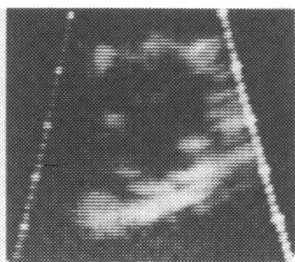

Fig. 4 (A) Parasternal short axis view diastolic frame of bicuspid aortic valve; $(B)$ parasternal short axis view systolic frame of non-stenotic bicuspid aortic valve. A, anterior aortic leaflet; $P$, posterior aortic leaflet.

leaflets were not often visualised. The angle formed by the imaginary medial extension of portions of the commissures imaged (Fig. 5C) often exceeded the commissural angle seen in the same patient, using the conventional parasternal short axis approach (Fig. 1B).

\section{Discussion}

A bicuspid aortic valve occurs in over $50 \%$ of patients with anatomically isolated aortic stenosis and in about $25 \%$ of patients with clinically pure aortic regurgitation. ${ }^{2}$ It is not known why one congenitally bicuspid valve becomes severely scarred, calcified, and stenotic; another only mildly scarred and regurgitant; another the site of infection; and another remains free of these complications. Therefore, it is probably advantageous for purposes of adequate follow-up and progress to detect all such valves as early in life as possible. Similarly, it would be very helpful to have a means of effectively excluding the presence of this common congenital cardiac defect.

Although the presence of an aortic ejection sound on auscultation is suggestive of a non-stenotic bicuspid aortic valve, ${ }^{17}$ such sounds may be heard in cases of aortic root dilatation of any aetiology, ${ }^{18}$ and when confined to the cardiac apex may easily be confused with late tricuspid valve closure. ${ }^{17}$

M-mode echocardiography has previously been reported as specific and reasonably sensitive in the detection of a bicuspid aortic valve. ${ }^{17} 19$ Use of the maximum eccentricity index for accurate indentification of abnormal valve anatomy has, however, recently been questioned. ${ }^{13}$ Transducer angulation cannot be precisely standardised, and transducer position and angulation on the chest wall may be dictated by differing thoracic shapes and altered orientation of cardiomediastinal structures. Because of this, the M-mode ultrasonic beam may intersect the bicuspid aortic valve cusps at successive locations, yielding widely variable values for the eccentricity index.

In cases of angiographic and/or surgically proved bicuspid aortic valve, technically optimal two dimensional echocardiographic parasternal short axis images at the level of the great arteries confirmed the presence of only two aortic leaflets opening and closing during the cardiac cycle in $65 \%(28 / 43), 2094 \%(33 / 35),{ }^{21} 100 \%$ $(13 / 13),{ }^{13}$ and $100 \%(11 / 11)^{14}$ of cases in four reported series. Data also suggest that diastolic images have greater sensitivity than systolic short axis images in the detection of aortic valve leaflet number. ${ }^{15}$ Systolic

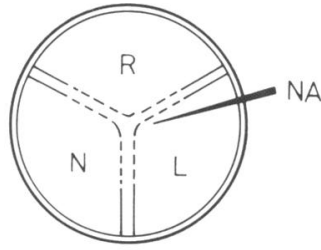

A $(n=8)$

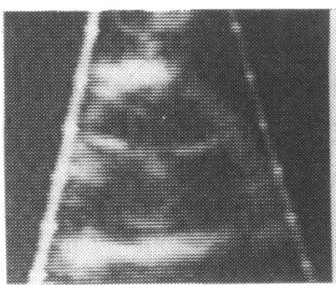

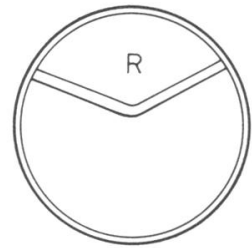

$\mathrm{B}$

$(n=5)$

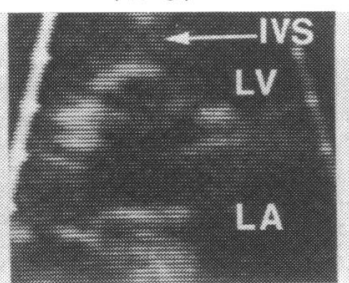

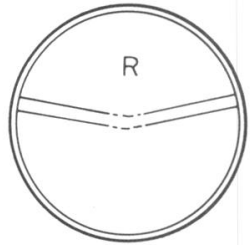

$(n=8)$

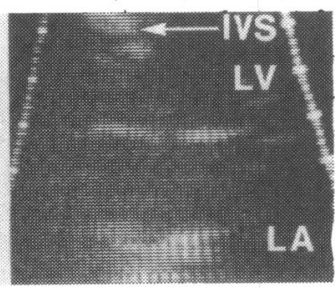

Fig. 5 (A) Aortic valve with " $Y$ " diastolic configuration as often incompletely imaged on anteriorly tilted apical four chamber view; (B) aortic valve with " $V$ " diastolic configuration on anteriorly tilted apical four chamber view; $(C)$ aortic valve with " $V$ " diastolic configuration as often incompletely imaged on anteriorly tilted apical four chamber view. $R$, right coronary cusp; $N$, noncoronary cusp; $L$, left coronary cusp; $N A$, noduli Arantii; IVS, interventricular septum; LA, left atrium; $L V$, left ventricle. 
valvular "doming" noted on two dimensional echocardiographic parasternal long axis imaging is present in only $46 \%(6 / 13),{ }^{13}(16 / 35)^{21}$ of angiographic and/or surgically proven cases of bicuspid aortic valve, and is confined to those cases with peak systolic transvalvular gradient greater than $33 \mathrm{mmHg}{ }^{13}$ The echocardiographic finding of valvular doming, moreover, is not specific for bicuspid aortic valve and is present in subjects with trileaflet aortic valvular stenosis and a significant transvalvular gradient. ${ }^{13}$ The usefulness of two dimensional echocardiography in the diagnosis of bicuspid aortic valve appears, therefore, to be dependent upon the frequency with which the commissural margins of the aortic valve cusps can be imaged during diastole using the parasternal short axis view.

Our results indicate that two dimensional echocardiographic recording of a parasternal short axis view at the level of the great arteries could indentify the probable number of valve leaflets using previously described echocardiographic criteria ${ }^{13} 15$ in only $27 \%$ of subjects. In addition, false positive and negative diagnoses of a bicuspid aortic valve have been reported previously using these criteria. ${ }^{13} 20$ Our figure of $27 \%$ is somewhat lower than that reported in the only other large series of prospectively examined subjects ${ }^{15}$ and may be because there are more subjects with densely calcified valves and/or more smokers among our patients. Age and smoking history were important variables affecting the technical quality of the parasternal short axis images obtained. This may be attributable to changes in the thoracic skeleton (calcification) and also to hyperinflation of the lungs (increased residual volume) which occurs with both age ${ }^{22}$ and cigarette smoking. ${ }^{23}$ Though the equipment used in this study produced a relatively good image, use of the latest generation ultrasonography devices, with higher frequency transducers, might well have resulted in greater sensitivity in the detection of aortic valve cusp morphology because of superior spatial resolution.

Systematic use of an anteriorly tilted apical four chamber view for aortic valve leaflet imaging was helpful in subjects who smoked and did not have a parasternal echocardiographic window which was technically adequate. This has not, however, been our experience when applying this technique to nonsmokers and subjects with an adequate parasternal echocardiographic window. In these subjects, a tangential section of the aortic root and cusps almost always results, which does not allow accurate identification of cusp morphology. For this reason, our study procedure systematically used the anteriorly tilted apical four chamber view only in subjects in whom a conventional parasternal short axis view at the level of the great arteries was not technically good enough. In these cases, perhaps, cardiac rotation, possibly related to chronic obstructive pulmonary disease, may have permitted adequate short axis imaging of the aortic root from the apical position.

In many other cases, the commissures between the non-coronary and left coronary cusps were not imaged. On the basis of previously reported pathological data, 211 the " $V$ " configuration formed by the remaining commissures and the noduli Arantii $(n=25)$ should be diagnostic of a tricuspid aortic valve, regardless of whether equal cusps and commissures, unequal cusps and equal commissures, or unequal cusps and commissures are present (Fig. 3A-C). In the latter case, it is essential to obtain an exact cross-section of the aortic root and to angle the beam slightly in all directions so as to find the minimal angle present between the two limbs of the imaged "V". If this is not done, this type of tricuspid aortic valve could be misdiagnosed as bicuspid. Since the raphe of a congenitally bicuspid valve rarely extends to the free margin of the conjoined cusp or to the cephalid level reached by the two true commissures, ${ }^{2} 20$ visualisation of the noduli Arantii, which are often echo reflective relative to the contiguous commissural margins of the aortic valve cusps, should also help in establishing the tricuspid nature of these valves.

Considering the high prevalence in our population of incomplete two dimensional echocardiographic aortic valve leaflet images, angiographic and/or pathological studies must be done to establish the correlation between these incomplete echocardiographic patterns and true aortic valve anatomy if two dimensional echocardiography is to have widespread application in both the detection and the exclusion of the congenital bicuspid aortic valve.

\section{References}

1 Koletsky S. Congenital bicuspid aortic valves. Arch Intern Med 1941; 67: 129-56.

2 Roberts WC. The congenitally bicuspid aortic valve. A study of 85 autopsy cases. Am F Cardiol 1970; 26: 72-83.

3 O'Rourke RA, Crawford MH. The systolic click-murmur syndrome: clinical recognition and management. Curr Probl Cardiol 1976; 1: 1-60.

4 Lewis T, Grant RT. Observations relating to subacute infective endocarditis. Heart 1923; 10: 21-99.

5 Edwards JE. The congenital bicuspid aortic valve. Circulation 1961; 23: 485-8.

6 Osler W. The bicuspid condition of the aortic valves. Trans Assoc Am Physicians 1886; 1: 185-92.

7 Fenoglio JJ Jr, McAllister HA Jr, DeCastro CM, Davia JE, Cheitlin MD. Congenital bicuspid aortic valve after age 20. Am F Cardiol 1979; 39: 164-9.

8 Smith DE, Matthews MB. Aortic valvular stenosis with coarction of the aorta with special reference to the development of aortic stenosis upon congenital bicuspid valves. Br Heart $\mathcal{F} 1955$; 17 : 198-206. 
9 Bacon APC, Matthews MB. Congenital bicuspid aortic valves and the aetiology of isolated aortic valvular stenosis. Qf Med 1959; 28: 545-60.

10 Campbell M. Calcific aortic stenosis and congenital bicuspid aortic valves. Br Heart $\mathcal{F} 1968$; 30: 606-16.

11 Roberts WC. The structure of the aortic valve in clinically isolated aortic stenosis; an autopsy study of 162 patients over 15 years of age. Circulation 1970; 42: 91-7.

12 Schlant RC. Calcific aortic stenosis. Am f Cardiol 1971; 27: 581-3.

13 Fowles RE, Martin RP, Abrams JM, Schapira JN, French JW, Popp RL. Two-dimensional echocardiographic features of bicuspid aortic valve. Chest 1979; 75: $434-40$.

14 Nanda NC, Gramiak R. Evaluation of bicuspid aortic valves by two-dimensional echocardiography (abstract). Am $\mathcal{F}$ Cardiol 1978; 41: 372.

15 Bansal RC, Tajik AJ, Seward JB, Offord KP. Feasibility of detailed two-dimensional echocardiographic examination in adults-prospective study of 200 patients. Mayo Clin Proc 1980; 55: 291-308.

16 Runyon RP, Haber A. Fundamentals of behavioral statistics. 3rd ed. Reading, Massachusetts: AddisonWesley, 1980.

17 Leech G, Mills P, Leatham A. The diagnosis of a nonstenotic bicuspid aortic valve. Br Heart $\mathcal{F}$ 1978; 40: 94150.

18 Craige E. Heart sounds. In: Braunwald E, ed. Heart disease-a textbook of cardiovascular medicine. Philadelphia: WB Saunders, 1980: 50.
19 Nanda NC, Gramiak R, Manning J, Mahoney EB, Lipchik EO, DeWeese JA. Echocardiographic recognition of the congenital bicuspid aortic valve. Circulation 1974; 49: 870-5.

20 Brandenburg RO Jr, Tajik AJ, Edwards WD, Reeder GS, Shub C, Seward JB. Accuracy of two-dimensional echocardiographic diagnosis of bicuspid aortic valve: echocardiographic-anatomic correlative study in 115 patients (abstract). Am f Cardiol 1982; 49: 1040.

21 Gondi B, Nanda NC. Two-dimensional echocardiography in bicuspid aortic valves: usefulness and limitations (abstract). Clin Res 1981; 29 (2): 197A.

22 Richards DW. Pulmonary changes due to aging. In: Fenn WO, Rahn H, eds. Handbook of physiology. A critical comprehensive presentation of physiologic knowledge and concepts. Section 3: Respiration Vol. 2. Washington, DC: American Physiologic Society, 1965: 1525-9.

23 Ingram RH Jr. Chronic bronchitis, emphysema and chronic airways obstruction. In: Isselbacher $\mathrm{KJ}$, Adams RD, Braunwald E, Petersdorf RG, Wilson JD, eds. Harrisons principles of internal medicine. 9th ed. New York: McGraw-Hill, 1980: 1239.

Requests for reprints to Dr Michael J Zema, Division of Cardiology, Department of Medicine, Brookhaven Memorial Hospital Medical Center, 101 Hospital Road, Patchogue, New York 11772, USA. 